\title{
Randomized Game Semantics for Semi-Fuzzy Quantifiers
}

\author{
Christian G. Fermüller and Christoph Roschger \\ Theory and Logic Group 185.2 \\ Vienna University of Technology
}

\begin{abstract}
Building on a generalization of classical Henkin-Hintikka evaluation games to Giles' game semantics for Lukasiewicz logic, a concept for evaluating (semi-)fuzzy quantifiers by randomizing the choice of witnessing elements is developed. In particular the adequateness of simple dialogue game rules for so-called proportionality quantifiers is shown.
\end{abstract}

\section{Introduction}

Fuzzy quantification combines the theory of generalized quantifiers [16] with degree based reasoning. As pointed out in Glöckner's monograph [11], there is what can be called an "embarrassment of richness" in modeling vague natural language quantifiers like many, few, about half, etc.: the space of candidates for truth functions is simply too complex and large, even if we restrict attention to monadic quantifiers, as we will do here. Glöckner addresses this challenge by focusing on semi-quantifiers first, i.e. quantifiers with classical (bi-valued) scope, and proposes an axiomatically defined scheme for lifting these to fully fuzzy quantification in a later step. Here we want to embed certain semi-fuzzy quantifiers into Łukasiewizc logic, which is not only one of most important formalisms of deductive (mathematical) fuzzy logics in the sense of Hájek $[12,13,5]$, but also allows for a particularly interesting semantic characterization in terms of dialogue games combined with bets on dispersive experiments, introduced by Giles $[10,9]$. In a sense, this reacts to the mentioned embarrassment of riches by suggesting a bottom-up approach: we start with a convincing and well explored semantic framework for standard first-order Lukasiewicz logic and extend it in a rather lean manner that remains close to its spirit.

Since we do not want to assume familiarity with Giles' game, we begin with the familiar Henkin-Hintikka evaluation game for classical logics and, unlike Giles, introduce many-valued games in reference to that classical game.

\section{Randomizing Henkin-Hintikka games}

As already shown by Hintikka [14], building on an idea of Henkin, the Tarskian notion of truth in a model can be characterized - and in fact generalized - by a two person game played on a first order formula with respect to a given model. We present the classical evaluation game in a slightly unusual terminology that will make the later transition to Giles' game more transparent. 
The $\mathcal{H}$-game. There are two players, say me and you, who can both act in the roles of either the attacker or the defender of a formula. The game is played with respect to a given classical first order interpretation $M$. To simplify the exposition we will identify domain elements with constants and restrict to a language without function symbols here. $M$ can thus be identified with an assignment of 0 (false) or 1 (true) to the variable free atoms of the language. By $v_{M}(F)$ we denote the truth value to which $F$ evaluates in $M$.

Every state of the game is determined by a sentence (closed formula) $F$ and a role assignment: either me or you act as the defender of $F$, the opponent player is the attacker. We will say that player $\mathbf{X}$ asserts $F$, if $\mathbf{X}$ is the defender of $F$. The game starts with my assertion of some formula and proceeds according to the following rules corresponding to the form of the currently considered formula.

$\left(R_{\wedge}\right)$ If I assert $F \wedge G$ then you attack by pointing either to the left or to the right subformula. I then have to assert $F$ or $G$, accordingly.

$\left(R_{\vee}\right)$ If I assert $F \vee G$ then I have to assert either $F$ or $G$ at my own choice.

$\left(R_{\neg}\right)$ If I assert $\neg F$ then our roles are switched and you have to assert $F$.

$\left(R_{\exists}\right)$ If I assert $\exists x F(x)$ then I have to pick a constant $c$ and assert $F(c)$.

$\left(R_{\forall}\right)$ If I assert $\forall x F(x)$ then you pick $c$ and I have to assert $F(c)$.

Note that we have stated the rules only for me in the role of the defender. However, because of $\left(R_{\neg}\right)$, the roles may switch. Since the rules for you asserting $F$ (and me attacking) are completely dual, they need not be stated explicitly. Moreover, we emphasize that the role assignment remains unchanged for all state transitions, except for the one explicitly triggered by $\left(R_{\neg}\right)$.

Once the game has arrived at an atomic state, i.e., at a state where either I or you assert an atomic formula $A$, I win (and you lose) if $v_{M}(A)=1$ and I lose (and you win) if $v_{M}(A)=0$. We call the game starting with my assertion of $F$ the $\mathcal{H}$-game for $F$ under $M$.

Theorem 1 (Hintikka) A sentence $F$ is true in an interpretation $M$ (in symbols: $v_{M}(F)=1$ ) iff I have a winning strategy in the $\mathcal{H}$-game for $F$ under $M$.

Our aim is to provide a similarly elegant characterization of graded truth for first order fuzzy logics. While game semantics can be generalized to cover a wide range of different many-valued logics (see $[2,4,8]$ ) we will stick here to infinite valued Łukasiewicz logic, which is one of, if not the most important example of a deductive fuzzy logic.

Łukasiewicz logic $\mathbf{E}$ provides two forms of conjunction: weak conjunction $(\wedge)$, and strong conjunction $(\&)$; negation $(\neg)$, implication $(\rightarrow)$, (weak) disjunction $(\vee)$, and standard quantifiers $(\forall$ and $\exists)$; specified semantically as follows:

$$
\begin{array}{lr}
v_{M}(F \wedge G)=\min \left(v_{M}(F), v_{M}(G)\right) & v_{M}(F \vee G)=\max \left(v_{M}(F), v_{M}(G)\right) \\
v_{M}(F \& G)=\max \left(0, v_{M}(F)+v_{M}(G)-1\right) & v_{M}(\perp)=0 \\
v_{M}(\neg F)=1-v_{M}(F) & v_{M}(F \rightarrow G)=\min \left(1,1-v_{M}(F)+v_{M}(G)\right) \\
v_{M}(\forall x F(x))=\inf _{c \in D}\left(v_{M}(G(c))\right) & v_{M}(\exists x F(x))=\sup _{c \in D}\left(v_{M}(G(c))\right)
\end{array}
$$

where $D$ is the domain of $M$ (which we identify with the set of constants). 
There are many good reasons to base $\mathbf{E}$ on the full syntax, as specified above. ${ }^{1}$ In particular this nicely fits the general theory of t-norm based fuzzy logics as introduced by Hájek $[12,13]$ and developed into a prolific subfield of mathematical logic by many researchers since, as witnessed by the recent

ook [3]. However, in the vast literature on fuzzy logic and on many-valued logics in general one frequently considers only $\wedge, \vee$, and $\neg$ as propositional connectives. We will call this fragment of $\mathbf{E}$, together with the standard quantifiers $(\forall, \exists)$, weak Eukasiewicz logic $\mathbf{E}^{w}$ here. The restrictions of $\mathbf{E}$ and $\mathbf{E}^{w}$ to the propositional part will be denoted by $\mathbf{E}_{p}$ and $\mathbf{E}_{p}^{w}$, respectively.

In order to transfer $\mathcal{H}$-games into a many-valued setting we borrow an idea of Giles [10,9] and reformulate the winning condition in a way that will lead to an interesting interpretation of intermediate truth values in terms of expected risks of payments. We conceive of the evaluation of the atomic formula $A$ at the final state of an $\mathcal{H}$-game as a (binary) experiment $\mathrm{E}_{A}$ that either fails, meaning $v_{M}(A)=0$, or succeeds, meaning $v_{M}(A)=1$. The experiment $\mathrm{E}_{\perp}$ always fails. Moreover, we stipulate that I have to pay $1 €$ to you if I lose the game. Hence winning strategies turn into strategies for avoiding payment. ${ }^{2}$ So far this just amounts to an alternative way to present the original game. The main innovation of Giles is to let the experiments $\mathrm{E}_{A}$ be dispersive. This means that $\mathrm{E}_{A}$ may show different results upon repetition, where the individual trials of the experiment are understood as independent events. (Of course, $\mathrm{E}_{\perp}$ remains nondispersive: it simply always fails.) The reader is invited to think about intended applications modeling vague language: while in concrete dialogues competent language users either (momentarily and provisionally) accept or don't accept grammatical utterances upon receiving them, vagueness results in a brittleness or dispersiveness of such highly context dependent decisions. (See, e.g, [17, 1].) In order to arrive at 'degrees of truth' for an atomic $A$ in such a model, one assumes that the dialogue partners associate a fixed success probability $\pi\left(\mathrm{E}_{A}\right)$ to the experiment $\mathrm{E}_{A}$, which may be thought of as (implicitly) answering the question "Do you accept $F$ (at this instance)?" By $\langle A\rangle=1-\pi\left(\mathrm{E}_{A}\right)$ we denote the risk associated with $A$, i.e., the expected (average) loss of money associated with an assertion of $A$. The function $\langle\cdot\rangle$ that maps each atomic sentence into a failure probability of the corresponding experiment is called risk value assignment. Note that risk value assignments are in 1-1-correspondence with (many-valued) interpretations via $\langle A\rangle_{M}=1-v_{M}(A)$.

For weak propositional Łukasiewicz logic $\mathbf{E}_{p}^{w}$ the characterization of graded truth via $\mathcal{H}$-games in this setting with randomized payoff is straightforward.

\footnotetext{
${ }^{1}$ Actually one can define all connectives of $\mathbf{E}$ from just $\rightarrow$ and $\perp$ or alternatively from $\&$ and $\perp$. But neither $\rightarrow$ nor \& can be defined from the remaining connectives.

${ }^{2}$ Note the asymmetry of the payoff scheme: even when the roles of attacker and defender are switched, it is me, not you, who has to pay upon losing the game. This is necessary to ensure that enforceable payments (inversely) correspond to truth values. Giles' extended game scenario allows one to restore perfect symmetry, as we will see in Section 3.
} 
Theorem $2 A \mathbf{L}_{p}^{w}$-sentence $F$ is evaluated to $v_{M}(F)=x$ in interpretation $M$ iff in the $\mathcal{H}$-game for $F$ under the corresponding risk value assignment $\langle\cdot\rangle_{M} I$ have a strategy that limits my expected risk to $(1-x) \in$, while you have a strategy that ensures that my expected risk is not below this value.

Proof. We use $\langle\mid G\rangle^{*}$ to denote my final expected risk in a game where I am defending and you are attacking $G$ assuming that we both play optimally by employing the usual min-max strategy. ${ }^{3}$ If I am the attacker and you are the defender of $G$ this value is denoted by $\langle G \mid\rangle^{*}$.

If $F$ is atomic then $\langle F \mid\rangle^{*}=1-\langle F\rangle_{M}$ and $\langle\mid F\rangle^{*}=\langle F\rangle_{M}$ and thus my risk is $v_{M}(F)$ in the former case and $1-v_{M}(F)$ in the latter case, as required. Otherwise we argue by induction on the complexity of $F$ that $\langle\mid F\rangle^{*}=1-v_{M}(F)$.

- If I assert $\neg G$, the game continues with your assertion of $G$ and $\langle\mid \neg G\rangle^{*}$ reduces to $\langle G \mid\rangle^{*}=1-\langle\mid G\rangle^{*}$, just like in the truth function for $\neg$.

- If I assert $G \vee H$ then I will pick $G$ or $H$ according to where my associated expected risk is smaller. Therefore $\langle\mid G \vee H\rangle^{*}=\min \left(\langle\mid G\rangle^{*},\langle\mid H\rangle^{*}\right)$, and thus $v_{M}(G \vee H)=\max \left(v_{M}(G), v_{M}(H)\right)=1-\min \left(1-\langle\mid G\rangle^{*}, 1-\langle\mid H\rangle^{*}\right)$.

- If I assert $G \wedge H$ then you will pick $G$ or $H$ according to where my associated risk, i.e., your expected gain, is higher. Therefore $\langle\mid G \wedge H\rangle^{*}=$ $\max \left(\langle\mid G\rangle^{*},\langle\mid H\rangle^{*}\right)$, corresponding to $v_{M}(G \wedge H)=\min \left(v_{M}(G), v_{M}(H)\right)$.

The cases where you defend and I attack $F$ are completely dual.

Note that we are only interested in expected payoffs. Since individual trials of experiments are independent events, truth functionality is preserved. Consider a game for $A \vee \neg A$ for example. While I will finally have to pay either $1 €$ or nothing, depending on the result of $\mathrm{E}_{A}$, my optimal expected loss under the risk value assignment corresponding to interpretation $M$ is $\min \left(\langle A\rangle_{M}, 1-\langle A\rangle_{M}\right) €$, which indeed amounts to $\left(1-v_{M}(A \vee \neg A)\right) \in$.

There is a slight complication in lifting Theorem 2 to the first order level: in an $[0,1]$-valued interpretation $M$ witnessing domain elements for quantified sentences may not exist. More precisely, we may have $v_{M}(\forall x F(x))<v_{M}(F(c))$ and $v_{M}(\exists x F(x))>v_{M}(F(c))$ for all constants $c$. For this reason we define the following general notion for games with randomized payoff (as in our new version of the $\mathcal{H}$-game, above, and in $\mathcal{G}$-games, introduced below).

Definition 1. A game with randomized payoff is $r$-valued for player $\mathbf{X}$ if, for every $\epsilon>0, \boldsymbol{X}$ has a strategy that guarantees that her expected loss is at most $(r+\epsilon) \in$, while her opponent has a strategy that ensures that the loss of $\boldsymbol{X}$ is at least $(r-\epsilon) €$. We call $r$ the risk for $\mathbf{X}$ in that game.

This notion allows us to state the generalization of Theorem 2 to $\mathbf{E}^{w}$ concisely:

\footnotetext{
${ }^{3}$ We assume the reader to be familiar with basic notions of game theory. Formally, we have described a finite zero-sum two person game in extended form with perfect information. Although the payoff is defined in terms of expected payments, probabilities do not enter the game itself. The min-max principle induces pure optimal strategies. I.e., mixed strategies are not needed to arrive at the (unique) equilibrium.
} 
Theorem $3 A \mathbf{L}^{w}$-sentence $F$ is evaluated to $v_{M}(F)=x$ in interpretation $M$ iff the $\mathcal{H}$-game for $F$ under risk value assignment $\langle\cdot\rangle_{M}$ is $(1-x)$-valued for me.

Proof. Building on the proof of Theorem 2, it only remains to consider the induction steps for quantified sentences:

- If I assert $\exists x F(x)$, then the game continues with my assertion of $F(c)$ for a constant $c$ picked by me in a manner that minimizes my risk. In fact, since there might be no domain element witnessing the infimum $v_{M}(\exists x F(x))=$ $\inf _{c \in D}\left(v_{M}(F(c))\right)$, we can only ensure that, for any given $\delta>0,\langle\mid \exists x F(x)\rangle^{*}=$ $\langle\mid F(c)\rangle^{*}=1-v_{M}(\exists x F(x))+\delta$.

- If I assert $\forall x F(x)$, the game continues with my assertion of $F(c)$, where $c$ is chosen by you to maximize my risk. Therefore, analogously, we obtain $\langle\mid \forall x F(x)\rangle^{*}=\langle\mid F(c)\rangle^{*}=1-v_{M}(\forall x F(x))-\delta$ for some $\delta>0$.

The cases where you are the defender of a quantified formula are dual.

Note that the value $\epsilon$ mentioned in Definition 1 does not directly correspond to $\delta$ as used in the above proof, but rather results from the accumulation of appropriate $\delta \mathrm{s}$. In any case, since our intended applications assume finite domains, we may from now on safely ignore the fact that, in general, truth values of statements involving quantifiers are only approximated by expected risk in concrete instances of a game. We nevertheless retain the notion of the value of a game, but could actually simplify Definition 1 by dropping all references to $\epsilon$.

\section{From $\mathcal{H}$-games to $\mathcal{G}$-games}

Already in the 1970s Robin Giles [10,9] introduced an evaluation game that was intended to provide 'tangible meaning' to reasoning about statements with dispersive semantic tests as they appear in physics. For the logical rules of his game Giles referred not to Henkin or Hintikka, but to Lorenzen's dialogue game semantics for intuitionistic logic [15]. In particular, (essentially) the following rule for implication was proposed:

$\left(R_{\rightarrow}\right)$ If I assert $F \rightarrow G$ then you may attack by asserting $F$, which obliges me to assert $G$. (Analogously if you assert $F \rightarrow G$.)

In contrast to $\mathcal{H}$-games, such a rule introduces game states, where more than one formula may be currently asserted by each of us. Since, in general, it matters whether we assert the same statement just once or more often, game states are now denoted as pairs of multisets of formulas. We call such games $\mathcal{G}$-games. A final state of a $\mathcal{G}$-game where $\left\{p_{1}, \ldots, p_{n}\right\}$ is the multiset of atomic assertions made by you and $\left\{q_{1}, \ldots, q_{m}\right\}$ is the multiset of atomic assertions made by me is denoted by

$$
\left[A_{1}, \ldots, A_{n} \mid B_{1}, \ldots, B_{m}\right] .
$$

Again we assume that a binary experiment $\mathrm{E}_{A}$ is associated with every atomic $A$ with corresponding risk $\langle A\rangle=1-\pi\left(\mathrm{E}_{A}\right)$. We now make payments fully dual and 
stipulate that I have to pay $1 €$ to you whenever an instance of an experiment corresponding to one of my atomic assertion fails, while you have to pay me $1 €$ for each instance of a failing experiment corresponding to one of your atomic assertions. We obtain the following value for the expected total amount of money (in $\in$ ) that I have to pay to you at the exhibited final state:

$$
\left\langle A_{1}, \ldots, A_{n} \mid B_{1}, \ldots, B_{m}\right\rangle=\sum_{1 \leq i \leq m}\left\langle B_{i}\right\rangle-\sum_{\leq j \leq n}\left\langle A_{j}\right\rangle .
$$

We call this value briefly my risk associated with that state. Note that the risk can be negative in $\mathcal{G}$-games, i.e., the risk values of the relevant propositions may be such that I expect a net payment by you to me.

Interestingly, the logical rules $\left(R_{\wedge}\right),\left(R_{\vee}\right),\left(R_{\forall}\right)$, and $\left(R_{\exists}\right)$ defined in Section 2 remain unchanged for $\mathcal{G}$-games. By adding the above implication rule $\left(R_{\rightarrow}\right)$ and defining $\neg F=(F \rightarrow \perp)$ we arrive at Giles' game for Eukasiewicz logic.

We like to point out that $\left(R_{\rightarrow}\right)$ contains a hidden principle of limited liability: the player opposing the defender of $F \rightarrow G$ may (instead of asserting $F$ in return for the opponent's assertion of $G$ ) explicitly choose not to attack $F \rightarrow G$ at all. This option results in a branching of the game tree. The state $[\Gamma \mid \Delta, F \rightarrow G]$, where $\Gamma$ and $\Delta$ are multisets of sentences asserted by you and me, respectively, and where the exhibited occurrence indicates that you currently refer to my assertion of $F \rightarrow G$, has the two possible successor states: $[F, \Gamma \mid \Delta, G]$ and $[\Gamma \mid \Delta]$. In the latter state you have chosen to limit your liability in the following sense. Attacking an opponent's assertion should never incur an expected (positive) loss, which were the case if the risk associated with asserting $F$ is higher than that for asserting $G$. In such cases a rational player in the attacking role will explicitly renounce an attack on $F \rightarrow G$. For all other logical connectives the principle is ensured by the fact that - in all games considered here-each occurrence of a formula can be attacked at most once. (The attacked occurrence is removed from the state in the transition to a corresponding successor state.)

Another form of the principle of limited liability can be considered for defending moves. In defending any sentence $F$, the defending player has to be able to hedge her (possible) loss associated with the assertions made in defense of $F$ to at most $1 €$. This is already the case for all logical rules considered so far. However, as shown in $[6,7]$, by making this principle explicit we arrive at a rule for strong conjunction, that is missing in Giles:

$\left(R_{\&}\right)$ If I assert $F \& G$, I have to assert either both $F$ and $G$, or assert $\perp$ instead.

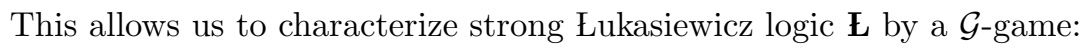

Theorem 4 (essentially Giles, but see also [7]) $A$ Ł-sentence $F$ is evaluated to $v_{M}(F)=x$ in interpretation $M$ iff the $\mathcal{G}$-game for $F$ under risk value assignment $\langle\cdot\rangle_{M}$ is $(1-x)$-valued for me.

\section{4 (Semi-)Fuzzy quantifiers and random witnesses}

As Glöckner emphasizes in his important monograph on fuzzy quantifiers [11], the very concept of generalized quantifiers over $[0,1]$ poses a challenge that may 
be termed an embarrassment of riches: even when we focus on logical quantifiers of type $\langle 1\rangle$, i.e., monadic quantifiers like $\exists$ and $\forall$, the space of possible truth functions is too large and complex to support the selection of plausible candidates for linguistically adequate models. ${ }^{4}$ We argue that a simple generalization of the game for $\mathbf{E}$, described in Section 3, allows one to address this challenge by singling out a lean class of quantifiers that nicely fits Giles' idea to provide "tangible meaning" to logical connectives in terms of bets on the results of dispersive experiments. Remember that the only difference between the rules $\left(R_{\forall}\right)$ and $\left(R_{E}\right)$ for defending assertions $\forall x F(x)$ and $\exists x F(x)$, respectively, is that either the defender or the attacker has to pick the constant $c$ that determines the new sentence $F(c)$ that remains to be defended. Because of the randomized setting of $\mathcal{G}$-games, the following rule for a new type of (unary) quantifier $\Pi$ seems natural:

$\left(R_{\Pi}\right)$ If I assert $\Pi x F(x)$ then I have to assert $F(c)$ for a randomly picked $c$.

The random choice refers to a uniform distribution of the domain. Note that, while all kinds of other forms of randomly picking domain elements might be considered in principle, we recall from the literature on generalized quantifiers (see, e.g., $[16,19])$ that a necessary condition for a quantifier to be called logical is the domain invariance of its semantics. ${ }^{5}$ As will get clear below, this is guaranteed for $\Pi$ (and for the quantifiers considered in Section 5) by insisting on random choices with respect to a uniform distribution.

While $\left(R_{\Pi}\right)$, arguably, makes sense for arbitrary $\mathbf{L}$-formulas $F$ in the scope of $\Pi$, we will view $\Pi$ as a semi-fuzzy quantifier and hence insist on classical formulas in its scope. To explain and motivate this design choice, we point out that Glöckner [11] responses to the above mentioned challenge ("too many" candidates for modeling fuzzy quantifiers) by focusing on semi-fuzzy quantifiers first and employing an axiomatically specified scheme for lifting those semifuzzy quantifiers to fully fuzzy quantifiers later. We follow this suggestion and enrich the language of $\mathbf{E}$ by distinguishing between classical (two-valued) and (possibly) fuzzy formulas already at the syntactic level. More formally, we specify the language for logic $\mathbf{E}(Q s)$, where $Q s$ is a list of (unary) quantifier symbols other than $\forall$ or $\exists$, as follows:

$$
\begin{aligned}
& \gamma::=\perp|\hat{P}(\boldsymbol{t})| \neg \gamma|(\gamma \vee \gamma)|(\gamma \wedge \gamma)|\forall v \gamma| \exists v \gamma \\
& \varphi::=\gamma|\tilde{P}(\boldsymbol{t})| \neg \varphi|(\varphi \vee \varphi)|(\varphi \wedge \varphi)|(\varphi \rightarrow \varphi)|(\varphi \& \varphi)|\forall v \varphi| \exists v \varphi \mid \mathrm{Q} v \gamma
\end{aligned}
$$

where $\hat{P}$ and $\tilde{P}$ are meta-variables for classical and for general (i.e., possible fuzzy) predicate symbols, respectively, $\mathrm{Q} \in Q s ; v$ is our meta-variable for object

\footnotetext{
${ }^{4}$ We use the term "linguistically adequate" here for vague determiners like "many", "few", "about half" etc. in the same sense as Glöckner [11], but want to emphasize that this amounts to a much less stringent criterion than empirical adequateness with respect to observable behavior of competent speakers as studied in (formal) semantics of natural language by linguists (see, e.g., [18]).

5 There is no agreement in the literature on when a generalized quantifier is to be called logical. However it is at least clear that invariance with respect to isomorphisms between domains is a necessary condition. See [16] for a discussion of this issue.
} 
variables; $\boldsymbol{t}$ denotes a sequence of terms, i.e. either object variable or a constant symbol, matching the arity of the preceding predicate symbol. Note the scope of the additional quantifiers from $Q s$ is always a classical formula. Otherwise the syntax is as for $\mathbf{E}$ itself.

The following notion supports a crisp specification of truth functions for semi-fuzzy quantifiers over finite interpretations.

Definition 2. Let $\hat{G}(x)$ be a classical formula and $v_{M}(\cdot)$ a corresponding evaluation function over the finite domain $D$. Then

$$
\operatorname{Prop}_{x} \hat{G}(x)=\frac{\sum_{c \in D} v_{M}(\hat{G}(c))}{|D|}
$$

Rule $\left(R_{\Pi}\right)$ matches the specification of $\Pi$ by $v_{M}(\Pi x F(x))=\operatorname{Prop}_{x} F(x)$.

Theorem $5 A \mathbf{L}(\Pi)$-sentence $F$ is evaluated to $v_{M}(F)=x$ in an interpretation $M$ iff the $\mathcal{G}$-game for $F$ augmented by rule $\left(R_{\Pi}\right)$ is $(1-x)$-valued for me under risk value assignment $\langle\cdot\rangle_{M}$.

Theorem 5 will turn out to be an instance of a more general result to be proved in the next section.

\section{$5 \quad$ Proportionality quantifiers}

Remember that in the context of our $\mathcal{G}$-games we have considered three types of challenges to the defender $\mathbf{X}$ of a quantified sentence $\mathrm{Q} x F(x)$. In each case $\mathbf{X}$ has to assert $F(c)$, but the constant (domain element) is either

(A) chosen by the attacker, or

(D) chosen by the defender, or

(R) chosen randomly.

We will speak of a challenge of type A, D or R, respectively. The need to variate these three challenges arises when we allow the defender (and possibly also the attacker) of $\mathrm{Q} x F(x)$ to bet either for or against $F(c)$. Betting for $F(c)$ simply means to assert $F(c)$, betting against $F(c)$ is equivalent to betting for $\neg F(c)$ and thus amounts to an assertion of $\perp$ in exchange for an assertion of $F(c)$ by the opposing player. Note that we can view this as follows: $\mathbf{X}$ pays $1 €$ for a betting ticket regarding $F(c)$ that entitles her to receive whatever payment by her opponent $\mathbf{Y}$ is due for Y's assertion of $F(c)$ according to the results of associated dispersive experiments made at the end of the game.

By allowing the players to choose between various successor states that result from an attack on $\mathrm{Q} x F(x)$ by certain numbers of bets for or against various instances of $F(x)$, where the constants replacing $x$ can be of type A, D, or R, we arrive at a rich set of possible quantifier rules. Here we will only investigate the family of proportionality quantifiers $\Pi_{k}^{m}$ specified by the following schematic game rule, where $\hat{F}$ is a classical formula: 
$\left(R_{\Pi_{m}^{k}}\right)$ If I assert $\Pi_{m}^{k} x \hat{F}(x)$ then $k+m$ constants are chosen randomly and I have to pick $k$ of those constants, say $c_{1}, \ldots, c_{k}$, and bet for $\hat{F}\left(c_{1}\right), \ldots, \hat{F}\left(c_{k}\right)$, while simultaneously betting against $\hat{F}\left(c_{1}^{\prime}\right), \ldots, \hat{F}\left(c_{m}^{\prime}\right)$, where $c_{1}^{\prime}, \ldots, c_{m}^{\prime}$ are the remaining $m$ random constants. (Likewise for your assertion of $\Pi_{m}^{k} x \hat{F}(x)$.)

Although not mentioned explicitly we emphasize that the principle of limited liability remains in place: after the constants are chosen, I may assert $\perp$ (i.e., agree to pay $1 \in$ ) instead of betting as indicated above.

We claim that this rule matches the extension of $\mathbf{E}$ to $\mathbf{E}\left(\Pi_{m}^{k}\right)$ by

$$
v_{M}\left(\Pi_{m}^{k} \hat{F}(x)\right)=\left(\begin{array}{c}
k+m \\
k
\end{array}\right)\left(\operatorname{Prop}_{x} \hat{F}(x)\right)^{k}\left(1-\operatorname{Prop}_{x} \hat{F}(x)\right)^{m} .
$$

Theorem $6 A \mathbf{L}\left(\Pi_{m}^{k}\right)$-sentence $F$ is evaluated to $v_{M}(F)=x$ in interpretation $M$ iff the $\mathcal{G}$-game for $F$ augmented by rule $\left(R_{\Pi_{m}^{k}}\right)$ is $(1-x)$-valued for me. under risk value assignment $\langle\cdot\rangle_{M}$.

Proof. Relative to the proof of Theorem 4 (see $[10,9,7]$ ) we only have to consider states of the form $\left[\Gamma \mid \Delta, \Pi_{m}^{k} x \hat{F}(x)\right]$. (I.e, we only consider my assertions of proportionally quantified sentences. The case for your assertions of $\Pi_{m}^{k} x \hat{F}(x)$ is dual.) For my enforceable risk at such a state we have

$$
\left\langle\Gamma \mid \Delta, \Pi_{m}^{k} x \hat{F}(x)\right\rangle^{*}=\langle\Gamma \mid \Delta\rangle^{*}+\left\langle\mid \Pi_{m}^{k} x \hat{F}(x)\right\rangle^{*}
$$

and it remains to show that my optimal way to reduce the exhibited quantified formula to instances as required by rule $\left(R_{\Pi_{m}^{k}}\right)$ results in a risk that corresponds to the specified truth function. In order to do so remember that the principle of limited liability is in place. Moreover remember that $\hat{F}$ is classical. This means that I either finally have to pay $1 €$ for my assertion of $\Pi_{m}^{k} x \hat{F}(x)$ or do not have to pay anything at all for it. The latter is only the case if all my bets for $\hat{F}\left(c_{1}\right), \ldots, \hat{F}\left(c_{k}\right)$, as well as all my bets against $\hat{F}\left(c_{1}^{\prime}\right), \ldots, \hat{F}\left(c_{m}^{\prime}\right)$, for $c_{1}, \ldots, c_{k}$, $c_{1}^{\prime}, \ldots, c_{m}^{\prime}$ as specified in rule $\left(R_{\Pi_{m}^{k}}\right)$ succeed. The probability that this event obtains, i.e., the inverse of my associated risk, is readily calculated to be

$$
\left(\begin{array}{c}
k+m \\
k
\end{array}\right) p^{k}(1-p)^{m}
$$

where $p=\operatorname{Prop}_{x} \hat{F}(x)$, which matches the relevant truth function in $\mathbf{E}\left(\Pi_{m}^{k}\right)$.

\section{Conclusion}

We have extended Giles' game based semantics for Łukasiewicz logic by considering randomly chosen domain elements as witnessing constants for certain instances of (semi-fuzzily) quantified statements. This allows one to specify the meaning of certain semi-fuzzy quantifiers in a manner that nicely fits the spirit of Giles' dialogue and betting game approach to approximate reasoning. 
This short introduction into that topic is not the place to discuss the employment of corresponding quantifiers to model vague natural language quantifiers like most, few, or about about half. Here we rather have to confine ourselves to the remark that, in order to arrive at linguistically adequate models, one should move from monadic to binary quantifiers and moreover combine appropriate game based definitions with mechanisms that incorporate context dependent parameters. Together with other relevant questions, like lifting to fully fuzzy quantifiers, axiomatization, complexity, the relation to other forms of randomized and game based semantics, etc., this is a topic for future work.

Acknowledgments. This work has been supported by the ESF/Austrian Science Foundation (FWF), Grant 1143-G15 (LogICCC/LoMoReVI).

\section{References}

1. C. Barker. The dynamics of vagueness. Linguistics and Philosophy, 25(1):1-36, 2002.

2. A. Ciabattoni, C.G. Fermüller, and G. Metcalfe. Uniform rules and dialogue games for fuzzy logics. In Logic for Programming, Artificial Intelligence, and Reasoning, volume 3452, pages 496-510. Springer, 2005.

3. P. Cintula, P. Hájek, and C. Noguera, editors. Handbook of Mathematical Fuzzy Logic. College Publications, 2011.

4. P. Cintula and O. Majer. Towards evaluation games for fuzzy logics. In Ondrej Majer, Ahti-Veikko Pietarinen, and Tero Tulenheimo, editors, Games: Unifying Logic, Language, and Philosophy, pages 117-138. Springer, 2009.

5. F. Esteva, L. Godo, P. Hájek, and M. Navara. Residuated fuzzy logics with an involutive negation. Archive for Mathematical Logic, 39:103-124, 2000.

6. C.G. Fermüller. Revisiting Giles's game. In O. Majer, A.-V. Pietarinen, and T. Tulenheimo, editors, Games: Unifying Logic, Language, and Philosophy, Logic, Epistemology, and the Unity of Science, pages 209-227. Springer, 2009.

7. C.G. Fermüller and G. Metcalfe. Giles's game and the proof theory of tukasiewicz logic. Studia Logica, 92(1):27-61, 2009.

8. C.G. Fermüller and C. Roschger. From games to truth functions: A generalization of Giles's game. submitted, see http://www.logic .at/staff/roschger/mvl_ dgames_preprint.pdf.

9. R. Giles. A non-classical logic for physics. Studia Logica, 33(4):397-415, 1974.

10. R. Giles. A non-classical logic for physics. In R. Wojcicki and G. Malinkowski, editors, Selected Papers on Eukasiewicz Sentential Calculi, pages 13-51. Polish Academy of Sciences, 1977.

11. I. Glöckner. Fuzzy quantifiers: a computational theory, volume 193 of Studies in Fuzziness and Soft Computing. Springer Verlag, 2006.

12. P. Hájek. Metamathematics of Fuzzy Logic. Kluwer Academic Publishers, 2001.

13. P. Hájek. What is mathematical fuzzy logic. Fuzzy Sets and Systems, 157(157):597603, 2006.

14. J. Hintikka. Logic, language-games and information: Kantian themes in the philosophy of logic. Clarendon Press Oxford, 1973.

15. P. Lorenzen. Logik und Agon. In Atti Congr. Internaz. di Filosofia, pages 187-194. Sansoni, 1960

16. S. Peters and D. Westerståhl. Quantifiers in language and logic. Oxford University Press, USA, 2006.

17. S. Shapiro. Vagueness in context. Oxford University Press, USA, 2006.

18. S. Solt. Vagueness in quantity: Two case studies from a linguistic perspective. In P. Cintula, C. Fermüller, L. Godo, and P. Hájek, editors, Reasoning under vagueness - logical, philosophical and linguistic perspectives, Studies in Logic. College Publications, to appear.

19. J. van Benthem. Questions about quantifiers. Journal of Symbolic Logic, 49(2):443466, 1984. 